\title{
Better tired than lost: turtle ant trail networks favor coherence over shortest paths
}

\author{
Arjun Chandrasekhar ${ }^{1}$, James A. R. Marshall ${ }^{2}$, Cortnea Austin ${ }^{3}$, Saket \\ Navlakha*4 $^{* 4}$ and Deborah M. Gordon*5 \\ ${ }^{1}$ Department of Computer Science, University of Pittsburgh, Pittsburgh, PA, \\ 15260 \\ ${ }^{2}$ Department of Computer Science, University of Sheffield, Sheffield, UK \\ ${ }^{3}$ Central Washington University, Ellensburg, WA 98926 \\ ${ }^{4}$ Simons Center for Quantitative Biology, Cold Spring Harbor Laboratory, \\ Cold Spring Harbor, NY, 11724 \\ ${ }^{5}$ Department of Biology, Stanford University, Stanford CA 94305
}

\begin{abstract}
Creating a routing backbone is a fundamental problem in both biology and engineering. The routing backbone of arboreal turtle ants (Cephalotes goniodontus) connects many nests and food sources using trail pheromone deposited by ants as they walk. Unlike species that forage on the ground, the trail networks of arboreal ants are constrained by the vegetation. We examined what objectives turtle ant networks meet by comparing the observed ant trail networks with networks of random, hypothetical trails in the same surrounding vegetation and with trails optimized for each objective. The ants' trails minimized the number of nodes traversed, reducing the opportunity for ants to get lost at each node, and favored nodes with 3D configurations most likely to be reinforced by pheromone, thus keeping the ants together on the same trail. Rather than finding the shortest path, turtle ant trail networks take advantage of natural variation in the environment to favor coherence, keeping the ants together on the trails.
\end{abstract}

Keywords: Cephalotes, ant trail network, routing networks, foraging, distributed algorithms, search, exploration, shortest path, spanning tree

\section{Introduction}

Many engineered systems rely on a backbone routing network, whose goal is to ensure that any two entities or devices on the network can communicate through some path $[1,2$,

*To whom correspondence should be addressed: navlakha@cshl.edu, dmgordon@ stanford.edu 
bioRxiv preprint doi: https://doi.org/10.1101/714410; this version posted July 12, 2020. The copyright holder for this preprint (which was not certified by peer review) is the author/funder, who has granted bioRxiv a license to display the preprint in perpetuity. It is made available under aCC-BY-NC-ND 4.0 International license.

3]. Some biological systems, such as neural arbors [4], plant arbors [5], and slime molds [6], also use routing networks to transmit information and nutrients. Effective design of routing networks depends on the physical environment, because variation in the environment can affect the accuracy and rate of communication in both engineered [7, 8, 9] and evolved natural networks $[10,11,12,13]$. The environment influences how the system chooses search strategies, prioritizes competing objectives, and coordinates its local decisions. For example, wireless networks operating in difficult to reach environments may use different routing strategies to minimize energy consumption of devices [7]; similarly, in bacterial navigation, chemicals appearing as localized pulses in the environment can affect gradient sensing and movement patterns [12].

The 14,000 species of ants have evolved diverse distributed routing algorithms to search for, obtain, and distribute resources $[14,15]$ in diverse environments [16, 17, 18, 19, 20]. Models of engineered routing networks inspired by ants often emphasize the goal of minimizing the distance traveled. Ant colony optimization (ACO), first proposed in 1991, loosely mimics ant behavior to solve combinatorial optimization problems, such as the traveling salesman [21, 22, 23] and other routing problems [24]. In ACO, individual ants each use a heuristic to construct candidate solutions, and then use pheromone to lead other ants towards better solutions. Recent advances improve ACO with techniques such as local search [25], cunning ants [26], and iterated ants [27].

A fascinating recent area of biological research examines the goals met by the trail networks of ants [20]. Studies of species that forage on a continuous 2D surface [18], including Pharaoh's ants [28], Argentine ants [17, 29, 30], leaf-cutter ants [16], army ants [31, 32], red wood ants [33], and meat ants [34, 35], show that ants use local chemical interactions to form trails [36, 37, 31], regulate traffic flow [38], search collectively [39], and form living bridges [40].

There are many objectives that an ant colony's trail network might meet, including minimizing costs in time and energy by reducing the distance traveled, keeping the ants together to form a coherent trail, being resilient to rupture, and searching effectively [41]. Ant species that forage and build trails on the ground have few constraints on trail geometry because their trails can form nodes and edges anywhere on the 2D plane. Prior work [42, 30, 41] showed that ground-living ants, such as red wood ants and Argentine ants, may minimize the distance traveled by forming trails with branch points that approximate 2D Steiner trees [17, 43, 44], trees of minimum total length that connect a given set of terminals, such as nests and food sources. However, minimizing distance may not be the only objective that ant trail networks attempt to optimize. Army ants sometimes link their bodies to form a bridge across gaps that shortens the path, but do not always make bridges because it reduces the number of available foragers. [45]. Meat ants form trail networks that link nests and trees as nodes, and their choices of which nodes are linked, as well as the direction and length of trails, suggest that robustness to the loss of a node is as important as minimizing the distance traveled [35, 34]. When given the choice of two paths of equal length, black garden ants prefer straight paths to curved paths [46].

Many ant species operate in 3D environments, such as arboreal ants that nest and forage in trees and bushes. Unlike species that have evolved to create graph structures in continuous space in a 2D plane, arboreal ants must form trails on a natural graph structure. They cannot form trails with nodes and edges at arbitrary locations; instead, they can use only the nodes and edges that are available to them. The arboreal turtle ant (Cephalotes goniodontus) nests and forages in the tree canopy of tropical forests [47]. A turtle ant colony creates a trail network in the vegetation that connects several nests, providing a routing backbone that must be maintained 
bioRxiv preprint doi: https://doi.org/10.1101/714410; this version posted July 12, 2020. The copyright holder for this preprint (which was not certified by peer review) is the author/funder, who has granted bioRxiv a license to display the preprint in perpetuity. It is made available under aCC-BY-NC-ND 4.0 International license.

to allow resources to be distributed throughout the colony $[48,49]$. The colony modifies the network from day to day, as it creates trails to temporary food sources, and occupies new nests and abandons old ones. Day to day changes in trails sometimes include small alternative paths, or loops, of which one is eventually chosen. The colony also repairs the backbone in response to frequent changes in the vegetation caused by plant growth and by ruptures made by wind or passing animals [49].

\section{Results}

\section{Candidate objectives}

We used data from field observations of the trail networks of turtle ants to test what objective functions these trails may be optimizing. We compared the observed networks with simulated random networks, to determine how well the observed networks meet three objectives.

1. Minimizing the distance traveled, which was measured as the average length of the edges in the trail network. This is equivalent to minimizing the total trail length for a fixed number of edges, and minimizes the costs in energy and time of building and maintaining the trail network. This distance, however, is not equivalent to the number of nodes traversed, as is often assumed in various optimization algorithms [50,24, 51, 52], including our previous work on turtle ants [53], because in the vegetation, the lengths of edges vary; the distance between one node and another ranges over 100-fold, from less than a centimeter to more than a meter (Table 1).

2. Minimizing the total number of nodes, which promotes the maintenance of a coherent trail by reducing opportunities for ants to get lost. The number of nodes was a count of all the nodes traversed in the trail network. In previous work, we used observational data to parameterize a model for how ants select a trajectory through a node to the next edge, based on the rate at which volatile pheromone is deposited [53]. Simulation results were consistent with field observations [48] indicating that at every node, there is a small probability that an ant may leave the trail to explore, by taking an edge that is not the one most strongly reinforced by pheromone [53]. Thus each node presents an opportunity for ants to get lost, and lost ants may lay pheromone trail that could lead other ants astray. Each node is also an opportunity to intersect with the network of a competing colony [54] in the same vegetation.

3. Maximizing the probability that successive ants take the same trajectory through a node. This objective contributes to the creation and maintenance of a coherent trail. The transition from one edge through a node to another edge is reinforced only when successive ants deposit pheromone along exactly the same trajectory through the node (Fig 1); the ants have short antennae that detect pheromone only locally. Nodes are variable 3D structures, ranging from a simple fork created by a branch in a plant, to a cluster of entwined vines and branches from different plants. We used a categorical index to estimate how many trajectories an ant could take from one edge through a node to another edge, and thus how likely a node is to be reinforced (Fig 1, details below). This index is not a measure of the biomechanical difficulty of a particular transition; ants do not seem deterred by features such as the 3D angle of two edges at a junction, except in very extreme cases [49]. We tested whether the ants' behavior reflected this estimate of the probability that successive ants take the same trajectory and thus reinforce it for the next ant. 
bioRxiv preprint doi: https://doi.org/10.1101/714410; this version posted July 12, 2020. The copyright holder for this preprint (which was not certified by peer review) is the author/funder, who has granted bioRxiv a license to display the preprint in perpetuity. It is made available under aCC-BY-NC-ND 4.0 International license.

This extends our previous work $[49,53]$ which did not take into account such variation among nodes, and did not examine how trails are selected from among the many available alternative networks.

We measured the use of nodes estimated to be likely to be reinforced as the average transition index of all transitions in the trail network. Each transition through a node in the vegetation (e.g., edge $u \rightarrow v$ through node $v$ to edge $v \rightarrow w$; Methods) was assigned a value of a transition index, based on a visual estimate of the number of different trajectories available for ants to traverse the node, drawing on previous observations of the flow of ants to different edges across a node (e.g., see Fig. 7 in [49]. Values ranged from 1, where we considered successive ants most likely to take and reinforce the same trajectory through the node because they traveled directly on a single plant, to 4 , where we considered successive ants to be least likely to take and reinforce the same trajectory through the node because there were many possible trajectories through a node that involved crossing from one plant to another (Fig 1). A particular node $v$ may have more than one transition index if there were many edges connected to that node. We did not measure pheromone deposition directly. Instead we tested whether the ants' choice of trajectory corresponded to our estimate of the trajectories most likely to be reinforced, by evaluating whether trails were likely to include transitions with a low transition index.

\section{Mapping and modeling turtle ant trail networks}

To examine what objectives are met by the ants' choice of paths within the vegetation (Fig 2A), we mapped the trail networks that connected the nests and naturally occurring, ephemeral food sources of three colonies (Tejon 189, Tejon 446, and Turtle Hill 460), for 10-15 days over the course of 6 weeks in 2018, and one colony (Tejon 500) for 5 days during 1 week in 2019, in a tropical dry forest at La Estación Biológica de Chamela in Jalisco, Mexico.

On each day, in each colony, we mapped the trail network, all paths taken by the ants that had a rate of flow ranging from 10 to 40 ants per 15 mins [49]. We identified each node or junction in the vegetation where an ant had a choice among more than one edge in the direction it was traveling. We measured the length of each edge, and assigned a transition index to each transition, from one edge through a node to another edge, by estimating how likely successive ants would be to take the same trajectory through the node and thus reinforce it with pheromone. To evaluate how the ants choose nodes and edges from the options provided by the surrounding vegetation, we also mapped all nodes, measured all edges, and assigned transition indices, for all possible paths up to five nodes away from each node used by the ants. A trail network on one day and some of the surrounding network of vegetation is illustrated in Fig $2 \mathrm{~B}-\mathrm{C}$.

We modeled the network of vegetation as a directed, weighted graph, $G=\left(V_{G}, E_{G}\right)$, where each junction forms a node, and edges represent stems or branches that connect one node to another. Edge weights correspond to physical length. We modeled transition indices by converting $G$ into its corresponding line graph (Methods). The nodes corresponding to the nests and food sources were designated as terminals. There were sometimes day to day changes in which terminals the ants' paths included in the network.

We compared the extent to which each observed network on a given day, connecting the terminals used on that day, optimized each of the 3 objectives, relative to a set of 100,000 random networks that connected the same terminals used in the observed networks. We compared the observed networks with random networks based on two null models (Methods): For the first null model, the random network generated on day $t$ modifies the random network generated 

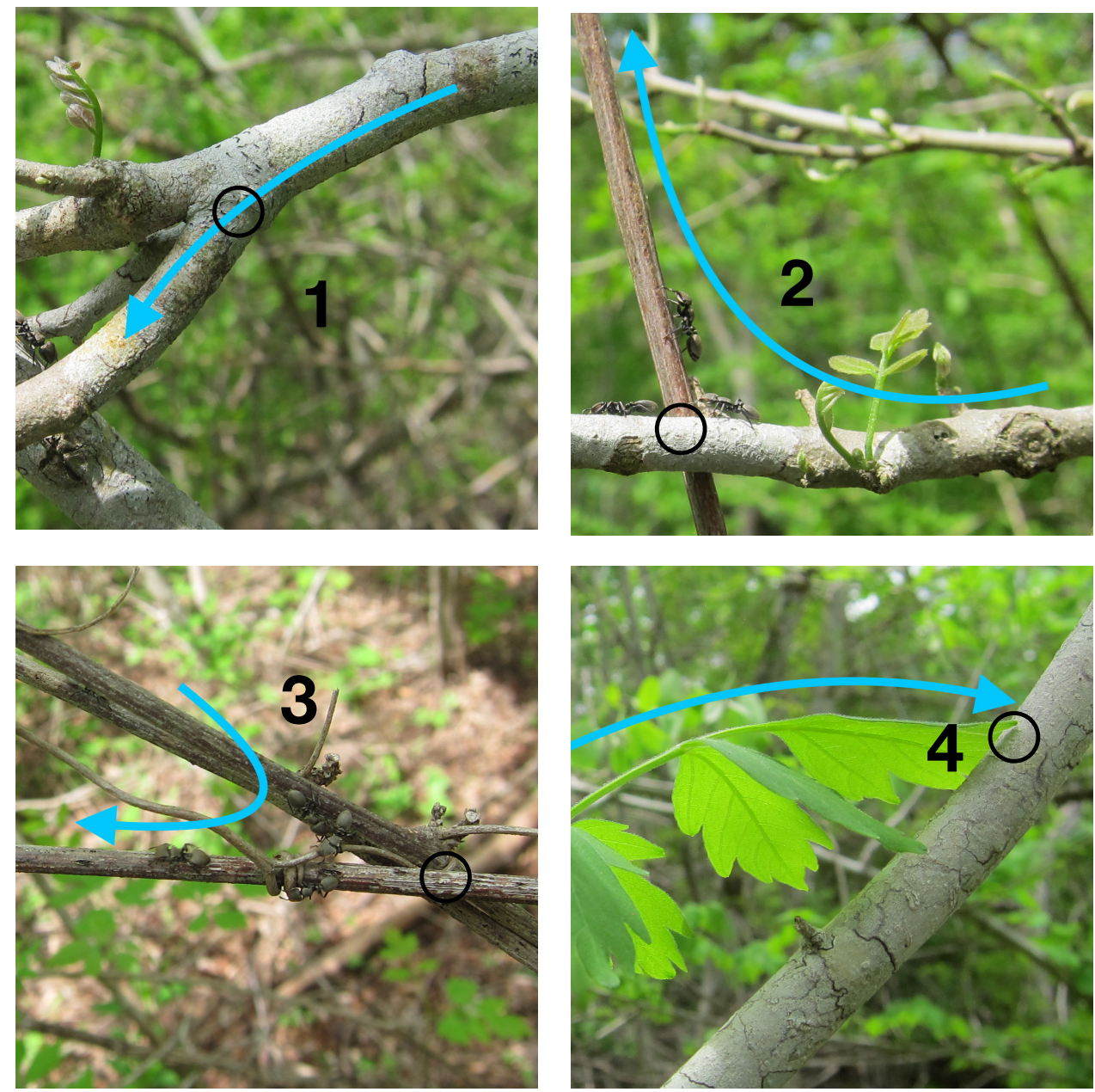

Figure 1: Transition indices. The photos show examples of nodes of each transition index (TI). The open black circle shows the node traversed. The blue arrow shows the transition, leading from the edge along which ants enter the node, through the node, toward the edge along which they exit. Each transition, from an edge to a node to another edge, was assigned a transition index with a value between 1 and 4 . A low transition index corresponds to a transition likely to be traversed using exactly the same trajectory by successive ants, and thus likely to be more rapidly reinforced. TI-1 (upper left): a node linking two edges on the same plant; in the example shown, all ants are likely to walk the same way across the top of the branch. TI-2 (upper right): a node that links one plant to another along a trajectory through a node that is likely to be the same for successive ants; in the example shown, most ants are likely to climb up the brown vine from the position shown by the ant approaching the junction from the left. TI-3 (lower left): a node that links one plant to another plant with more than one possible trajectory through the node; in the example shown, ants on the upper vine can reach the lower one either directly or by following the smaller vine that ants in the photo are using. TI-4 (lower right): a node that links one plant to another with many possible trajectories that are often changed by conditions; in the example shown, wind can easily move the leaf so that different ants reach the junction between the leaf and branch, at different places.

on day $t-1$ by removing paths to terminals not used on day $t$ and adding new random paths 


\section{A}

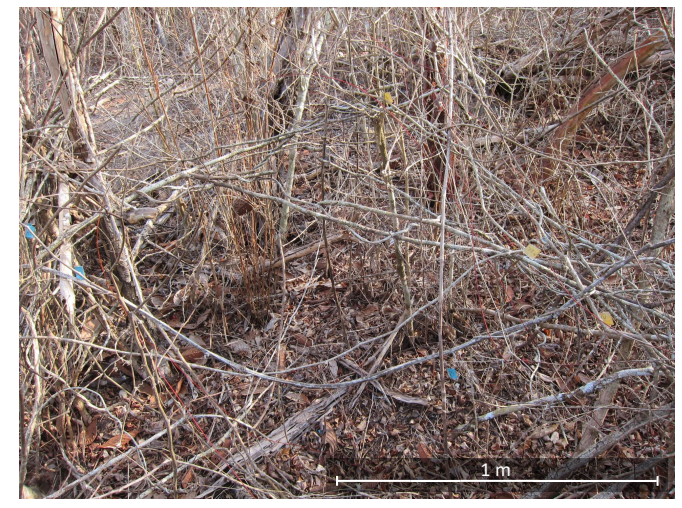

B

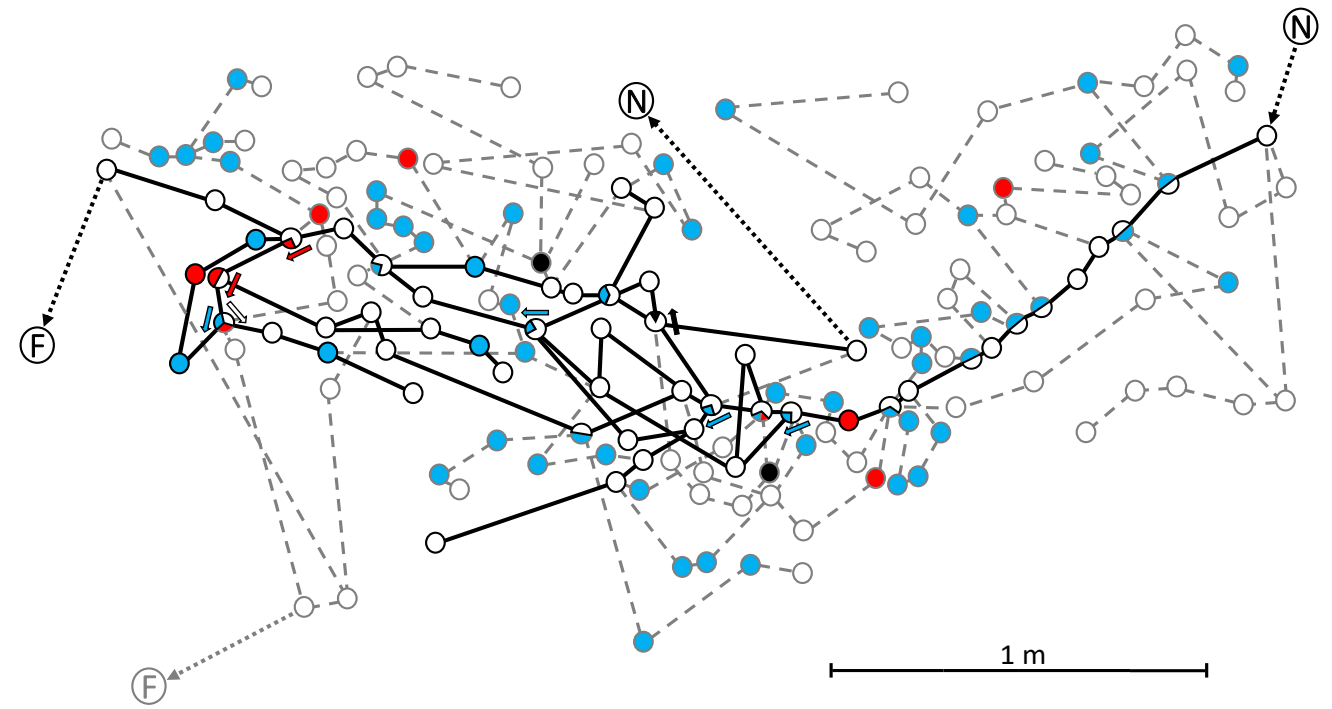

Figure 2: Used and available networks in the vegetation. A) Vegetation in which the trail network mapped in B was made, photographed in the dry season before the branches have leaves. B) Illustration of part of the trail network and network of surrounding vegetation for Tejon 189 on day 9. The figure shows 166 of the 217 nodes mapped in the surrounding vegetation. Edge lengths are scaled to measured distance, but actual location is not represented here. $\mathrm{N}$ represents a nest, F represents a food source. Circles represent nodes. Solid lines represent edges used on that day; dashed lines represent edges not used that day (Methods). The color of a node represents the transition index (TI) from the preceding edge to the following one: TI-1, open circles; TI-2, blue; TI-3, red; TI-4, black. At a node where there is a choice of more than one edge in the indicated direction, so that there could be more than one transition taken through a given node, a TI was assigned to each possible transition. For such nodes with more than one transition index, the TI is represented graphically with a pie chart, and arrows show which transition has the TI represented by the arrow's color. 


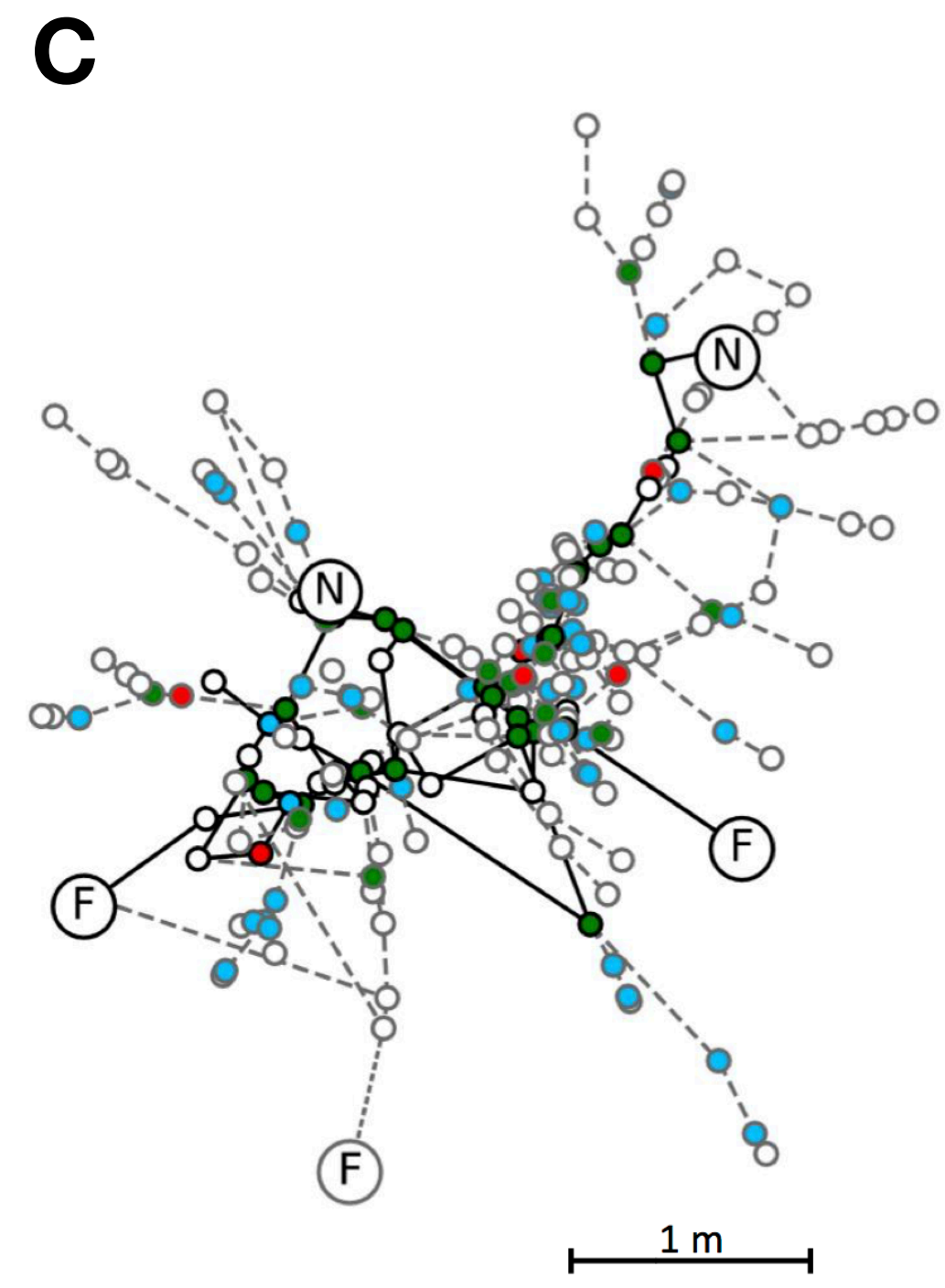

Figure 2: Used and available networks in the vegetation. C) Map of all 217 nodes for Tejon 189 on day 9. Symbols as in B. The large size of the network makes it difficult to show all TIs per node, so nodes with more than one TI are colored green.

networks are similar from day to day [49]. The second method generates a new random network from scratch each day, so each day is independent of the others.

To determine which of the three objectives the trail networks were most likely to optimize, we also compared the observed ant networks to non-random networks generated by heuristic algorithms that optimized each objective independently (Methods). To compare observed and random networks for each objective, we used a percentile measure (Methods). The percentile measure evaluates how much better the observed network does at optimizing each objective than random networks. The lower the percentile, the better the observed network optimized the objective, and a percentile of $50 \%$ means the observed network is equal to random networks.

To compare observed networks with optimized networks, we performed two steps. First, we computed the percentile of observed networks with respect to random networks, and the percentile of optimized networks with respect to random networks. Typically, optimized networks had a percentile of $0 \%$, but on some days, the percentile was slightly higher because the 
bioRxiv preprint doi: https://doi.org/10.1101/714410; this version posted July 12, 2020. The copyright holder for this preprint (which was not certified by peer review) is the author/funder, who has granted bioRxiv a license to display the preprint in perpetuity. It is made available under aCC-BY-NC-ND 4.0 International license.

\begin{tabular}{lcc|cc|cc|c}
\hline & \multicolumn{2}{c|}{ Edge Length (cm) } & \multicolumn{2}{c|}{ Total Nodes } & \multicolumn{2}{c}{ Transition Index } & Connectivity \\
& Avail & Used & Avail & Used & Avail & Used & Used \\
\hline Tejon 189 & 10698 & $20.64 \pm 6.17$ & 217 & $43.50 \pm 9.43$ & 1.60 & $1.31 \pm 0.06$ & $3.75 \pm 0.23$ \\
Tejon 446 & 7625 & $13.48 \pm 0.71$ & 196 & $35.91 \pm 4.06$ & 1.69 & $1.44 \pm 0.13$ & $4.00 \pm 0.24$ \\
Tejon 500 & 7341 & $26.20 \pm 2.43$ & 147 & $25.20 \pm 6.94$ & 1.88 & $1.60 \pm 0.12$ & $2.46 . \pm 0.34$ \\
Turtle Hill 460 & 14696 & $40.00 \pm 9.39$ & 202 & $54.20 \pm 18.23$ & 1.47 & $1.44 \pm 0.13$ & $4.50 \pm 0.92$ \\
\hline
\end{tabular}

Table 1: Comparison of trail networks used and available in the surrounding vegetation. Values for 'Available' are for the entire mapped vegetation network, including the nodes and edges that were used. Values for 'Used' are means \pm S.D., averaged over observation days, for the paths used in each colony's network ( $n=10$ for Tejon 189 and Turtle Hill 460, $n=5$ for Tejon 500, and $n=11$ for Tejon 446). Connectivity is a measure of the smallest number of nodes needed to get back to the trail used by the ants from an edge off the trail, averaged over all edges that lead off the trail used by the ants (Methods).

heuristic algorithm is not guaranteed to find the optimal network. Second, we compared the two percentiles for each objective to determine if the observed networks are more similar to random networks than to optimized networks.

\section{Turtle ant trail networks favor coherent trails over shortest paths}

The ants' networks optimized the maintenance of coherent trails. First, when we compared the observed networks with the day-to-day dependent random null model, there were significant differences in how well observed networks optimized each objective (Scheirer-Ray-Hare twofactor ANOVA df $2, H=46.918, p<0.001)$. Trail networks minimized average transition index (Conover test, $T=6.547, p<0.001$ ) and minimized the total number of nodes (Conover test, $T=8.691, p<0.001$ ), significantly more than they minimized the distance traveled (Fig 3A). There were no significant differences between the extent to which observed networks, compared to random networks, minimized the average transition index and the total number of nodes (Conover test, $T=2.144$, ns).

The observed networks were much more similar to the optimized networks in the extent to which they minimized nodes and transition index than average length (Fig 3A). The percentiles of observed versus random were: $3.24 \pm 8.67$ for total nodes, $7.75 \pm 19.76$ for TI, $49.11 \pm 44.13$ for average length. The percentiles of optimized versus random were: $0.11 \pm 0.27$ for total nodes, $1.35 \pm 1.76$ for TI, $0.02 \pm 0.03$ for average length. We compared these two percentiles to determine whether observed networks were more similar to optimized than they were to random (Methods). Observed networks were more similar to optimized than to random networks for total nodes (Wilcoxon signed ranks test, $n=36, W=6, p<0.001$ ) and average transition index (Wilcoxon signed ranks test, $n=36, W=76, p<0.001$ ) than to average length (Wilcoxon signed ranks test, $n=36, W=180, p<0.05)$.

Colonies did not differ overall in the how well the observed networks met the 3 objectives, relative to the random networks (S-Test, df 3, $H=2.974$, ns), but there was a significant objective x colony interaction ( $S$-test, df $2, H=20.973, p<0.01$ ), apparently due to differences among colonies in the local vegetation in which they traveled. Two of the colonies, Turtle Hill 460 and 
bioRxiv preprint doi: https://doi.org/10.1101/714410; this version posted July 12, 2020. The copyright holder for this preprint (which was not certified by peer review) is the author/funder, who has granted bioRxiv a license to display the preprint in perpetuity. It is made available under aCC-BY-NC-ND 4.0 International license.

Tejon 500, minimized total nodes, relative to random networks, significantly more than they minimized average transition index (Conover-Imam test [55, 56], T460, $T=3.965, p<0.001$; T500, 5.042, $p<0.001)$. Unlike the other 3 colonies, Turtle Hill 460 did not minimize average transition index significantly more than average length, relative to random networks (Conover test, $T=2.135, \mathrm{~ns}$ ), and Tejon 446 did not optimize total nodes significantly more than average length (Conover test, $T=1.285, \mathrm{~ns}$ )

Random networks with the same number of nodes as the observed network did not differ in total length from the observed network. We tested this to control for the confounding of total edge length and total number of nodes because they are correlated $(R=0.79)$, and to test which one is prioritized when the two objectives give different results. In all four colonies, the total length of observed networks was similar to that of the random networks with the same number of nodes: the percentiles were $28.87 \pm 35.17 \%$ for Tejon $189,39.39 \pm 17.27 \%$ for Tejon 446 , $31.87 \pm 37.16 \%$ for Turtle Hill 460, and $31.87 \pm 37.17 \%$ for Tejon 500 .

We also compared observed networks with random networks generated by the second null model in which random networks were generated independently for each day (Methods). The results were nearly identical: compared to random networks, the observed networks optimized total nodes and average transition index, but not total length (S1 Text)

\section{Turtle ant trail networks increase coherence over time}

From day to day, progressive changes in the trail networks of all four colonies tended to minimize the average transition index and the total number of nodes more, and more consistently, than they minimized average length (Fig 3B-F). Fig 3B shows an example of a day-to-day change that minimized both the number of nodes and average transition index. From day 9 to day 10, the network changed from the path shown in yellow to the path shown in blue, thus eliminating the 6 nodes circled, including 2 nodes with TI-2 and one node of TI-4, in favor of a path with nodes all of TI-1 (Fig 3B). Overall, trails in Tejon 189 (Fig 3C) consistently minimized the total number of nodes and average transition index but twice increased the average length (days 7 to 10 and 11 to 15). Similarly, in Tejon 446 (Fig 3D), trails progressively decreased the total number of nodes and average transition index, but increased average length from days 6 to 7 , 8 to 9 , and 11 to 13 . In Turtle Hill 460 (Fig 3E), the network consistently minimized the total number of nodes. Changes in the nests and food sources used led to new networks with lower transition indices (days 2 to 10). There was an initial decrease in average edge length (days 4 to 7), due to a rupture on day 6 of a node leading to a $95 \mathrm{~cm}$ edge (one of the longest edges we measured) rather than to a choice of shorter edges, and then the trails increased in average edge length (days 7 to 14). The networks of Tejon 500 (Fig 3F) consistently minimized total nodes and average transition index, but not average length. Overall, these day-to-day changes indicate that turtle ant networks do not consistently minimize the distance traveled.

Using the day-to-day independent null model (Methods), the comparisons also showed that progressive changes over time minimized number of nodes and transition indices rather than distance traveled (S1 Fig).

\section{Turtle ants form loops to promote coherence}

Turtle ants form loops in their paths, consisting of small, temporary alternative paths with the same start and end points, and over time, all but one of these paths tends to be pruned 
A

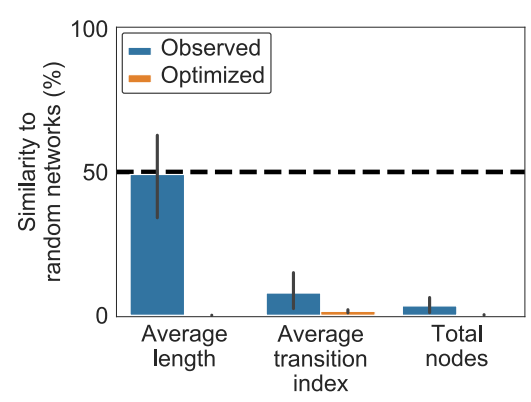

C Tejon 189

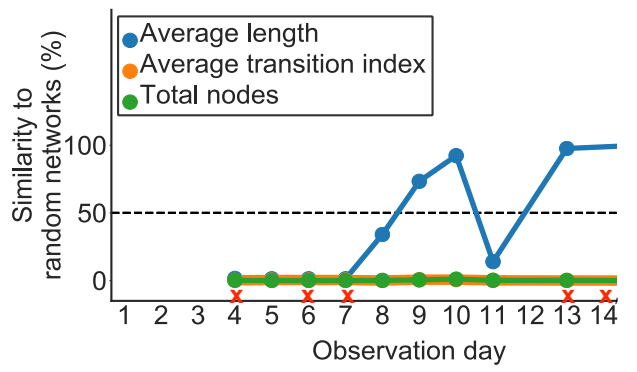

E Turtle Hill 460

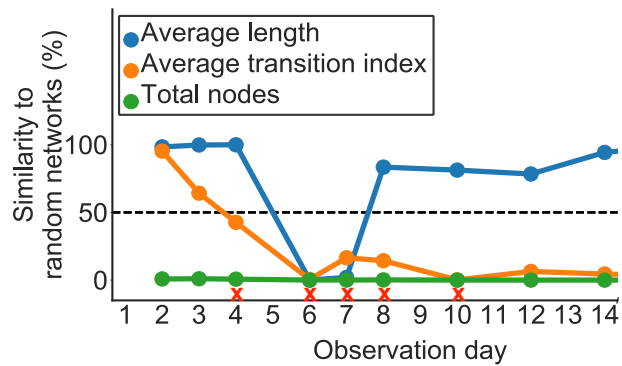

B

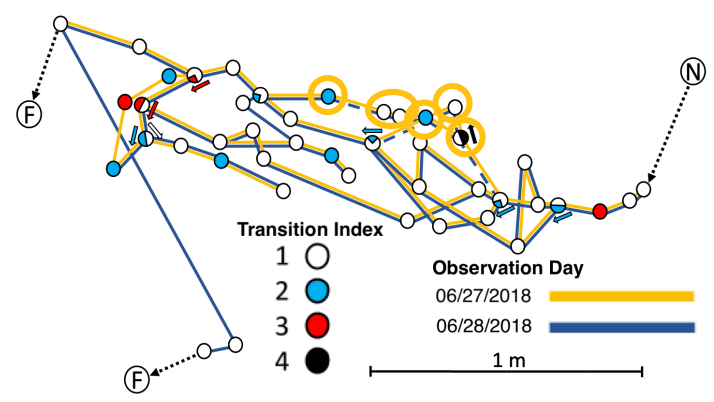

D Tejon 446

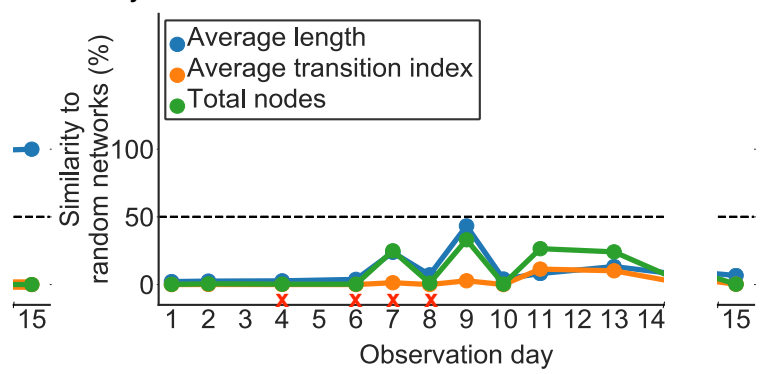

F Tejon 500

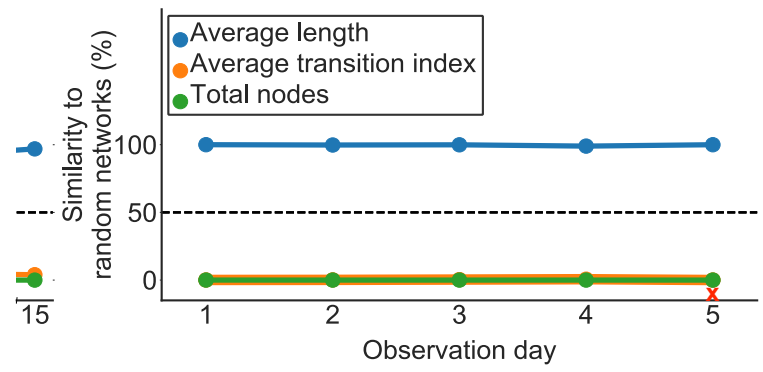

Figure 3: Comparison of random, optimized, and observed networks. A) Similarity of random, optimized, and observed networks measured as mean percentile (Methods). A percentile of 50 (dashed line) indicates that the observed or optimized network optimized the objective to the same extent as the average random network; the lower the percentile, the better the network optimized the objective compared to random networks. Error bars show standard errors of the mean. B) Day to day change in trails in Tejon 189, showing a change that decreased both number of nodes and average transition index. Symbols for transition index are the same as in Fig 2B: TI-1, open circles; TI-2, blue; TI-3, red; TI-4, black. Solid yellow lines show trails used on day 9; solid blue lines show trails used on day 10; yellow circles and dashed blue lines show trails linking the six nodes that were used on day 9 but not on day 10. C-F) Day to day changes in mean percentile for each objective. A red ' $X$ ' indicates one or more ruptured edges on that day. In $\mathrm{C}-\mathrm{E}$, the break in the $\mathrm{x}$-axis between observations days 14 and 15 represents an interval of about a month.

away [49]. Loops are often considered to decrease the efficiency of routing networks [2, 3], but may increase robustness by offering alternative paths if links are broken. Here we hypothesize that loops may occur because trails tend to form along the most rapidly reinforced nodes, and some sequence of rapidly reinforced nodes may naturally form a cycle in the graph.

Compared to available loops in the vegetation with the same start and end points (Methods), 
bioRxiv preprint doi: https://doi.org/10.1101/714410; this version posted July 12, 2020. The copyright holder for this preprint (which was not certified by peer review) is the author/funder, who has granted bioRxiv a license to display the preprint in perpetuity. It is made available under aCC-BY-NC-ND 4.0 International license.

the observed loops tended to use nodes with lower transition indices, and fewer nodes. We compared the centered ranks (Methods) of the average transition index, number of nodes, and average edge length, of paths connecting the same two start and end points on each trail in observed and available loops. The more negative the value of the centered rank, the lower the average transition index an observed path was compared to all of the random paths within the available vegetation with the same start and end points. The mean (SD) of the centered rank in observed loops for average transition index was -2.13(4.13); for number of nodes was $-2.51(4.09)$ and for average edge length was 0.60(2.95); in all cases, significantly different from 0 (T-Test, $|T|>3.2, p<0.01$ ). These results suggest that loop formation in trails may occur as a consequence of selecting trails that have lower transition indices, thus promoting coherence.

\section{Discussion}

The trail networks of arboreal ants link nests and find temporary food sources in changing vegetation. They respond to dynamic environments by maintaining the coherence of their trails. Minimizing the transition index and minimizing the number of nodes contribute to coherence because they both diminish the risk of losing ants from the trail. Like water flowing over a rocky stream bed, turtle ants tend to use trails most conducive to the flow of ants. When the physical configuration of a node provides a trajectory that successive ants are likely to follow, the chances are reduced that turtle ants wander off the path and lay pheromone trails that can lead other ants also to leave the trail.

Trail networks must also balance the tradeoff between exploration and coherence. Exploration is necessary for the colony to construct trails [57, 30, 41, 17, 40, 38], search for new resources $[58,59,60,61,62,39]$ and repair breaks $[49,53,34]$, and the connectivity of the vegetation (Table 1) influences the probability that ants that leave the trail will return to another node on the trail. There appears to be a small probability of exploration at each node $[49,53]$, so the probability of leaving the trail accumulates with more nodes traversed. Thus, minimizing the number of nodes traversed reduces opportunities for the ants to get lost.

Nodes with transition indices of 1 keep the trail on the same plant [49]. The vines and trees of the tropical dry forest tend to have long internode distances to reach the sunlight at the edge of the canopy [63]. By staying on the same plant, ants are led to resources at the edge of the canopy, such as flowers that provide nectar.

Minimizing distance traveled is often considered the main objective in engineered routing algorithms and ant colony optimization [24, 22, 23], since rapid communication is often needed between any two nodes in the network. We showed that the trail networks of turtle ants instead optimize the maintenance of a coherent trail by minimizing the total number of nodes, and by minimizing the average transition index; optimizing the latter objective, we show, is NPcomplete (Methods). In previous work [53], we proposed a model for the algorithm used to maintain and repair networks. This algorithm did not distinguish between minimizing the number of nodes and the distance traveled, since each edge had equal length. Our results here show that further work is needed to develop an algorithm that captures the role of physical variation in the environment.

Our results here suggest that, as in engineered networks [64, 65], the cost of including a node in the turtle ant network may vary among nodes, because whether other ants follow an exploring 
bioRxiv preprint doi: https://doi.org/10.1101/714410; this version posted July 12, 2020. The copyright holder for this preprint (which was not certified by peer review) is the author/funder, who has granted bioRxiv a license to display the preprint in perpetuity. It is made available under aCC-BY-NC-ND 4.0 International license.

ant that leaves the trail at that node depends on the node's physical configuration. The costs of additional nodes include the loss of ants from the trail, and the pursuit of fruitless paths, which may detract from the colony's ability to distribute resources among its many nests, and make fewer ants available to recruit effectively when a new food source is discovered. In addition, each node provides opportunities for encounters with other, competing species traveling in the same vegetation [66, 67, 68, 54]. Further work is needed to examine variation among colonies [69] in how well they minimize the number of nodes, to learn how selection may shape the process that determines how a trail network is constructed and maintained.

We compared observed ant networks with two kinds of random networks, first that modified the previous day's trail, and second with random networks generated from scratch each day, which sampled more broadly from the space of all possible networks. Both models gave similar results: both performed significantly worse than the ant networks for minimizing the total number of nodes and average TI. This suggests that the ant networks are very far from being random, and that there are an extremely large number of sub-optimal networks in the vegetation. Moreover, the ant networks, using only local cues and de-centralized decision-making, are very close to performing as well as heuristically optimized algorithms that use centralized, global information.

Are there useful applications of the principle that variation in the environment provides useful constraints on routing network design? Evolved algorithms operating in the natural world can use structure in the environment to enhance coordination among distributed agents [70]. In engineering, taking into account the physical structure of the environment may improve the design of routing algorithms $[71,72,73,74,75]$, for example, by reducing the search space of possible routing paths and steering network construction away from parts of the terrain that are difficult to reach. This could be beneficial in applications such as robot swarms, where distributed agents must coordinate in complex environments using a communication backbone to explore new terrain, exploit of temporary resources, and maintain security against intruders [54].

\section{Materials and Methods}

\section{Observation of trail networks}

The trail networks of four turtle ant colonies (Turtle Hill 460, Tejon 446, Tejon 189, and Tejon 500) were observed between 06/20/2018 and 06/28/2019 at La Estación Biológica de Chamela in Jalisco, Mexico. Tejon 189 was observed for 10 days from 6/22/208-6/28/2018 and again on 07/01/2018, 7/04/2018 and 8/3/2018. Tejon 446 was observed for 11 days on 6/20/20186/28/2018 and again on 07/01/2018, 07/04/2018, and 08/03/2018; and Turtle Hill 460 was observed for 10 days from 06/21/2018-06/28/2018 and again on 07/02/2018, 07/05/2018, and $08 / 03 / 2018$. Tejon 500 was observed for 5 days between $06 / 24 / 2019$ and $06 / 28 / 2019$. Not every network was observed on each day. The colonies appeared to be of about the same size, based on observations in comparison with previous work in which size was estimated with mark-recapture measures [48], but here we did not measure colony size.

The numbers of observation days were 10 for Tejon 189 and Turtle Hill 460, 11 for Tejon 446 , and 5 for Tejon 500, for a total of $10+10+11+5=36$ observed networks. We considered how each of these 36 networks optimize 3 objective measures for a total of $36 \times 3=108$ comparisons. 
Networks were mapped using the same methods as in previous work [49], using visual inspection of the paths the ants took through the vegetation at a rate of at least 10 ants per 15 min. To track changes in the path and terminals used each day, each node was assigned a number, and enough nodes were marked, with small labels or stickers on a dead end branch near the node, to identify all same nodes the next day.

The assignment of the transition index was made using the following criteria:

- TI-1 (Fig 1A): a node linking two edges on the same plant.

- TI-2 (Fig 1B): a node that links one plant to another along a trajectory through a node that is likely to be the same for successive ants.

- TI-3 (Fig 1C): a node that links one plant to another plant with more than one possible trajectory through the node.

- TI-4 (Fig 1D): a node that links one plant to another with many possible trajectories that is often changed by conditions such as the wind.

In each day of observation, we recorded which edges and nodes were used by the ants. As observed previously [49], each colony's trail network changed which nodes it used from day to day, although each day's path conserved some parts of the previous day's. In the course of the observations reported here, the path of the ants never went outside the range of the 5 nodes around the trail that were originally mapped. The number of nodes observed in each network is shown in Table 1.

\section{Assigning directionality to edges}

Experiments with marked ants show that ants tend to use particular routes from a nest [48], and tend not to turn around on the trail. To account for this, for all edges we defined a direction relative to one terminal; the outbound direction went away from it, and the inbound direction went towards it. We restricted the analysis to paths that proceed in the outbound direction.

\section{Modeling the transition index as a line graph}

The transition index of a node is an estimate of the probability that successive ants will take the same trajectory from an edge $(u, v)$ through node $v$ to edge $(v, w)$. Thus a transition index involving node $v$ is assigned to a particular incoming edge used to reach $v$ and a particular edge used to leave $v$.

To model the transition index, we used the line graph of $G$, where $L_{G}=\left(V_{L}, E_{L}\right)$. The nodes $V_{L}=E_{G}$, and the edges $E_{L}=\left\{((x, y),(y, z)) \mid(x, y),(y, z) \in E_{G}\right\}$. The line graph creates a node for each edge in $G$ and connects two nodes if they correspond to two adjacent edges in $G$. Every edge in the line graph denotes a transition in the original graph; traversing the edge $((u, v),(v, w)) \in L_{G}$ corresponds to starting at $u$, going to $v$, crossing the junction at $v$, and then going to $w$. We assigned every edge in $L$ a transition index. Fig 4 illustrates this process. 
A Original Graph

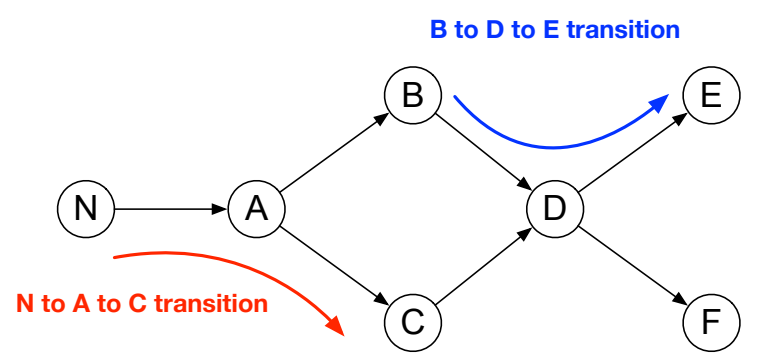

B Line Graph

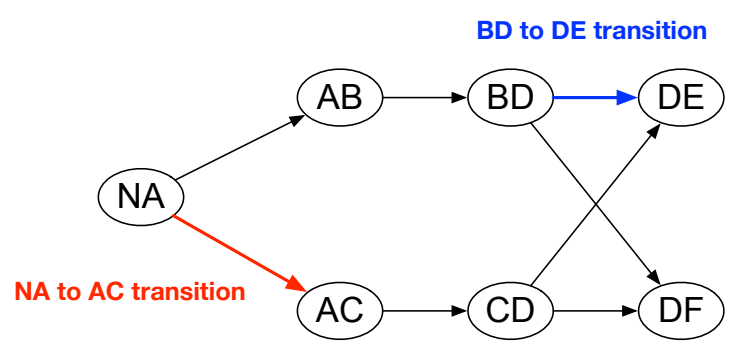

Figure 4: Converting the network to the line graph. A) Original graph. Nodes correspond to junctions in the vegetation, and edges correspond to links, such as a branch or stem, between junctions. B) Line graph. Every edge in the original graph has a corresponding node in the line graph. Two nodes are connected in the line graph if they correspond to two adjacent edges in the original graph. Transitions in the original graph used in this example are highlighted in red and blue.

\section{Generating random trail networks}

We compared the average edge length, total number of nodes, and average transition index in observed and random trail networks. The random networks were simulated in the graph made from the map of the paths used by ants and the surrounding vegetation, using the number of nodes, edge lengths and transition indices measured in the vegetation. The random trail networks may include loops, as did the observed networks.

We used two methods to generate random trail networks. The first null model, called the day-to-day dependent model, starts with an initial random network, and then randomly modifies it from day to day to connect the same terminals, nests, and food sources that were used by the turtle ants. Results are reported in the text. The second null model, called the day-today independent model, generates a new random network from scratch every day. Results are reported in S1 text.

For the day-to-day dependent model, we start by creating a random network that connects all of the terminals (nests and food sources) observed to be used on the first observation day. This initial network is different for each simulation. To generate a random network for each subsequent day, we modify the previous day's trail network to reflect any changes to the set of terminals used by the turtle ants. If a new terminal is included in the observed network, we add a path going from that terminal to the existing random network. If a terminal is no longer used in the observed network, we remove the path going from the terminal to the random network.

Formally, we start by generating an initial network $G_{1}$ for the first observation day:

1. Input: Graph $G=\left(V_{G}, E_{G}\right)$, terminals $X \subseteq V_{G}$.

2. Create an empty graph $G_{1}$; add a random terminal $x \in X$ to $G_{1}$.

3. Choose a random terminal $x^{\prime} \in X$ that has not already been added to $G_{1}$.

4. Perform a random walk on $G$ that starts at $x^{\prime}$ and stops when it touches any node $n$ in $G_{1}$.

5. Add all edges and nodes touched by the random walk to $G_{1}$. 
6. Repeat steps $3-5$ until all terminals have been added to $G_{1}$.

Let $G_{t}$ be the trail network generated randomly for day $t$. We now describe the process of constructing network $G_{t+1}$ for day $t+1$. First, we define the following term: when we add a terminal $x^{\prime}$ to the trail network, we perform a random walk that starts at $x^{\prime}$ and stops when it touches a node $e$ that is already in the network; we call $e$ an endpoint.

To construct $G_{t+1}$ we use the following procedure:

1. For each terminal $x_{i}$ that was used on day $t$ and not used on day $t+1$, let $W_{i}$ be the random walk through the vegetation that started at $x_{i}$ and was originally used to connect $x_{i}$ to the existing network (either on day $t$ or an earlier day). Let $e$ be the first endpoint that appears in $W_{i}$.

2. Remove all nodes in $W_{i}$ that are never touched after $e$ is touched for the first time.

3. For each terminal $x_{j}$ that was used on day $t+1$ and not on day $t$ : perform a random walk that starts at $x_{j}$ and stops when it touches any node $u$ in $G_{t+1}$. Add all nodes and edges touched by the random walk to $G_{t+1}$.

After randomly generating an initial network $G_{1}$ for day $t=1$, we use the above procedure to generate a network for day 2; and then continue this process for all observation days. This procedure reflects the observation that that turtle ant trails use similar paths from day to day. We ran 100,000 simulations in which we generated a different initial network.

In S1 Text, we present results from the second null model in which each random network is generated independently of the random network from the previous day. On each day, we use the same 6 step procedure that was used to create an initial network, to create a new network from scratch using the same terminals as the observed network.

\section{Approximating optimal networks}

We evaluated networks generated by heuristic algorithms designed to optimize each objective. Finding the network that optimizes total nodes is a variant of the classic Steiner tree problem (an NP-complete problem) [64, 65], and so we applied a standard approximation algorithm [76]. To optimize average length, we used the following algorithm:

1. Input: Graph $G=\left(V_{G}, E_{G}\right)$ and terminals $X \subseteq V_{G}$

2. Create an empty graph $T$.

3. Add edges $T$ in order of increasing length until $T$ contains all terminals $X$, and all terminals are in the same connected component.

4. Remove all nodes and edges not part of the same connected component as the terminals $X$

5. Remove edges from $T$ in decreasing order of length; only remove edges that decrease the average length without disconnecting $T$

To optimize average transition index, we use a nearly identical algorithm, but we add and remove edges in order of transition index rather than length. 
bioRxiv preprint doi: https://doi.org/10.1101/714410; this version posted July 12, 2020. The copyright holder for this preprint (which was not certified by peer review) is the author/funder, who has granted bioRxiv a license to display the preprint in perpetuity. It is made available under aCC-BY-NC-ND 4.0 International license.

\section{Comparing observed and random networks}

For each of the 4 colonies (Tejon 189, Tejon 446, Turtle Hill 460, and Tejon 500), and for each objective (average length, total nodes, and average transition index), we computed the similarity of each observed network to 100,000 random networks that connected the same set of terminals, using Equation (1).

To compare the observed and random networks, we computed for each network the following three objectives: 1) Average edge length: the average length of all edges in the network; 2) Total number of nodes: the total number of nodes in the network; and 3) Average transition index: the average transition index over all transitions in the network.

For each objective, we computed a value of $\varphi_{\text {ants }}$ for the observed network and $\varphi_{1}, \varphi_{2}, \ldots, \varphi_{n}$ for the $n$ random networks. We measured the similarity between the observed network and the random networks for that objective as the percentage of random networks that have a lower value for the objective:

$$
100 \times \frac{\left|\left\{\varphi_{i} \mid \varphi_{i} \leq \varphi_{\mathrm{ants}}\right\}\right|}{n} .
$$

An observed network is most similar to a random network when its percentile is $50 \%$. The closer the percentile is to 0 , the better the observed network optimized the objective, and the closer the percentile is to 100 , the better the random network optimized the objective. This percentile-based approach is unit-less, making it possible to compare performance for objectives that differ in the range of values.

Statistical comparison of observed and random networks. We used the non-parametric Scheirer-Ray-Hare two-factor ANOVA test $[77,78]$ to test for effects of objective, colony, and the colony $\mathrm{x}$ objective interaction on the percentiles of the observed networks compared to random networks. We then applied post hoc Conover-Iman non-parametric tests [55, 56], using a Bonferroni correction for multiple comparisons, with a significance threshold of $p=0.05$.

\section{Comparing observed and optimized networks}

To compare how similar observed and random networks were to optimized networks, we first computed the percentile of observed networks with respect to random networks, and the percentile of optimized networks with respect to random networks. We then tested if the observed networks were significantly more similar to random networks than to optimized networks. For each objective, we evaluated the difference between the observed network and random network as: 0.50 - (the observed network's percentile), and the difference between the observed network and the optimized network as: (the observed network's percentile) (the optimized network's percentile). We found this difference for each of the 36 observed networks and each of the 3 objectives, for a total 108 differences for each comparison. We tested whether observed were more similar to random than to optimized networks using a Wilcoxon's signed rank test for each objective.

\section{Comparing total length in observed and random networks}

Because the total number of nodes and the total edge length are correlated, we did not simultaneously compare total length and total number of nodes when comparing random and 
observed trail networks. Instead, we tested whether there was a difference in total length between observed and random trail networks with the same number of nodes. For each observation of one colony on one day, we found all the randomly generated networks that had the same number of nodes as that observed network. The total number of random networks and the number of percentile comparisons were: Tejon 189, 879, 10; Tejon 446, 7461, 11; Turtle Hill, 1607,6 ; Tejon $500,36,2$. We then found the total lengths of all of these networks, and used Equation (1) to evaluate how well each observed network optimized total length compared to random networks with the same number of nodes.

\section{Loops}

We compared the average transition index and number of nodes in observed loops with loops that were available in the surrounding vegetation. A loop was defined as two or more outbound paths that start and end at the same source and target nodes. For each observed loop, we computed all possible paths in the vegetation between the corresponding source and target nodes. We ranked all paths based on the average transition index of the path, and compared this to the rank of the average transition index of the paths used by the ants. We repeated the same measure for total number of nodes and average edge length.

We computed the centered rank of each path used by the ants as follows: We ranked $n$ available paths in the vegetation, by the average transition index of nodes in the path, total number of nodes in the path, or average length of edges in the path. The ranks of the paths are $1,2,3, \ldots n$, and the median rank is $r_{m}=(n+1) / 2$. For each observed path, we computed its centered rank by subtracting the median rank from that path's rank. Using average transition index as an example, the centered rank is 0 when the observed loop had the same average transition index as the median random loop, negative when the observed loop has a lower average transition index, and positive when the observed loop has a higher average transition index. We performed a similar analysis for the total number of nodes and average edge length.

\section{Connectivity}

To calculate the connectivity of the vegetation (Table 1), we estimated how many nodes are required for an ant that leaves the trail to return to the trail. For each edge $(u, v)$ connecting a node on the trail to a node off the trail, we found the length, in number of nodes, of the path with fewest nodes from $v$ back to a node on the trail. We measured connectivity as the smallest number of nodes in a path back to the trail, averaged over all edges $(u, v)$ leading off the trail on all days.

\section{Optimizing transition index is NP-complete}

Here we show that the problem of constructing the trail network that connects a given set of terminals while minimizing the average transition index of the network is NP-complete. To do this, we start by showing that finding the minimum average-weight path between two vertices in a graph is NP-complete. In this problem, we are given a graph $G=\left(V_{G}, E_{G}\right)$ and two vertices $u, v \in V$. The goal is to find a path $\mathcal{P}=\left[\left(u, r_{1}\right),\left(r_{1}, r_{2}\right), \ldots,\left(r_{k}, r_{k+1}\right),\left(r_{k+1}, v\right)\right]$ that minimizes the average edge length: $\frac{1}{|\mathcal{P}|} \sum_{e \in \mathcal{P}} w(e)$, where $w(e)$ defines the length of edge $e$. 
This problem differs from the classic shortest path problem, which seeks a path with minimal total edge length.

The standard method for considering the complexity class of an optimization problem is to consider the equivalent decision version of the problem: given a graph $G=\left(V_{G}, E_{G}\right)$, two vertices $u, v \in V_{G}$, and an integer $k$, we ask: is there a path from $u$ to $v$ whose average weight is $\leq k$ ?

\section{Lemma 1. Finding the minimum average-weight path is NP-Complete.}

Proof. First, we show that this problem is in the class NP. If we are given a path from $u$ to $v$, we can verify that the path is a valid $u-v$ path, and that the average edge weight is $\leq k$. This certificate will clearly be of polynomial length, and we can verify that it is correct in polynomial time.

Next, we show that the problem is NP-hard. We proceed via reduction from the Hamiltonian path problem, which is NP-Complete. Given a directed graph $G=\left(V_{G}, E_{G}\right)$, the directed Hamiltonian path problem seeks a path that touches every vertex $v \in V_{G}$ exactly once. We construct a graph $G^{\prime}$ as follows: $G^{\prime}$ contains all of the vertices in $G$ along with two additional vertices, $s$ and $t$. We assign a weight of 1 to all of the original edges in $G$. We add a directed edge from $s$ to every vertex $v \in V_{G}$, and we add a directed edge from every vertex $v \in V_{G}$ to $t$. We assign a weight of 2 to all edges that include either $s$ or $t$.

The smallest possible average edge weight for any path from $s$ to $t$ is $\frac{3+\left|V_{G}\right|}{\left|V_{G}\right|+1}$, and such a path exists if and only if $G$ has a directed Hamiltonian path.

The reduction requires adding 2 new nodes, and $2|V|$ new edges to $G$. Thus the reduction clearly takes only polynomial time.

We now use Lemma 1 to show that optimizing average transition index is NP-Complete. Let $L=\left(V_{L}, E_{L}\right)$ be a graph whose edge weights correspond to transition indices, which we represent using the line graph (Fig 4). Given a set of terminals $X \subseteq V_{L}$, we seek to find a subtree $F_{X} \subseteq L$ that minimizes the average value of all edge weights in $F_{X}$. We require that $F_{X}$ be connected without cycles to represent the fact that cycles in turtle ant trails are typically pruned.

Once again we consider the decision problem: given a line network $L=\left(V_{L}, E_{L}\right)$, a set of terminals $X$, and an an integer $k$, does $L$ contain a subtree $F_{X}$ whose average weight is $\leq k$ ?

\section{Lemma 2. Finding the subtree $F_{X}$ that optimizes average transition index is NP-Complete.}

Proof. First, we prove that the problem is in NP. Given $V_{L}, X, k$, if we are presented with a subgraph $F_{X}$ as a certificate, we can verify that $F_{X}$ is a valid solution. We check that $F_{X}$ is valid subgraph, that $F_{X}$ is connected, that $F_{X}$ contains every terminal node $X$, and that the average weight of the edges in $F$ is $\leq k$. Clearly the size of $F_{X}$ is polynomially bounded, and we can verify that $F_{X}$ is a valid solution in polynomial time.

To show that the problem is NP-hard, we proceed via reduction from the minimum averageweight path problem above. Given $G$ and vertices $u$ and $v$, we simply treat $G$ as the line graph $(L)$, and designate terminals $X=\{u, v\}$; this means $F_{X}$ is simply a $u$-v path. We seek the trail that optimizes the average transition index between terminals $u$ and $v$. By construction, this is the minimum average-weight path from $u$ to $v$ in the line graph, meaning there is a subgraph $F_{X}$ with average weight of $\leq k$ if and only if the original graph has a $u-v$ path with average edge weight $\leq k$. Further, the reduction clearly takes only polynomial time. 
The classic Steiner tree problem, in which the objective is to minimize total length, is known to be fixed-parameter tractable in the number of terminals [79]; that is, if the number of terminals is held constant then the problem can be solved in polynomial time. By contrast, optimizing transition index is not fixed-parameter tractable for the number of terminals; our reduction shows that optimizing average transition index is NP-hard even if we restrict the number of terminals to 2 . Thus, optimizing average transition index is more complex than even the classic NP-complete Steiner tree problem.

\section{Acknowledgements}

We thank Ibai Zabaleta and especially Taggert Butterfield for assistance in the field; and Daniel Beck, Katharine Renton, and the staff at the Estación Biológica de Chamela for their help. We are grateful for the comments of anonymous reviewers of a previous version of the manuscript.

\section{Funding Disclosure}

The work was supported by a grant from the CISCO Research Fund to DMG, a grant from the National Science Foundation under award CAREER DBI-1846554 to SN, a grant from the National Science Foundation under award 1559447 to Daniel Beck that supported field work for CA, funding from Chapman Foundations Management, LLC, to AC, and by the European Research Council (ERC) under the European Union's Horizon 2020 research and innovation programme (grant agreement number 647704 to JARM).

\section{Author Contributions}

AC: data analysis, computational modeling, manuscript preparation. JARM: computational modeling, manuscript preparation. CA: data collection, manuscript preparation. SN: data analysis, computational modeling, manuscript preparation. DMG: data collection, data analysis, computational modeling, and manuscript preparation.

\section{Competing Interests}

None of the authors have competing interests.

\section{Data Availability Statement}

All data collected and analyzed will be archived at Stanford Digital Repository at the Stanford Libraries. Python code is available at: https://github.com/DiODeProject/ SteinerAnts. 


\section{References}

[1] Lynch, N. A. Distributed Algorithms. San Francisco, CA, USA: Morgan Kaufmann Publishers Inc., 1996.

[2] Alwan, H. and Agarwal, A. "A survey on fault tolerant routing techniques in wireless sensor networks”. In: 2009 Third International Conference on Sensor Technologies and Applications. IEEE. 2009, pp. 366-371.

[3] Chouikhi, S., El Korbi, I., Ghamri-Doudane, Y., and Saidane, L. A. “A survey on fault tolerance in small and large scale wireless sensor networks". In: Computer Communications 69 (2015), pp. 22-37.

[4] Chandrasekhar, A. and Navlakha, S. "Neural arbors are Pareto optimal". In: Proc. Biol. Sci. 286.1902 (2019), p. 20182727.

[5] Conn, A., Pedmale, U. V., Chory, J., and Navlakha, S. "High-resolution laser scanning reveals plant architectures that reflect universal network design principles". In: Cell systems 5.1 (2017), pp. 53-62.

[6] Tero, A., Takagi, S., Saigusa, T., Ito, K., Bebber, D. P., Fricker, M. D., Yumiki, K., Kobayashi, R., and Nakagaki, T. "Rules for biologically inspired adaptive network design”. In: Science 327.5964 (2010), pp. 439-442.

[7] Fei, Z., Li, B., Yang, S., Xing, C., Chen, H., and Hanzo, L. "A survey of multi-objective optimization in wireless sensor networks: Metrics, algorithms, and open problems". In: IEEE Communications Surveys \& Tutorials 19.1 (2016), pp. 550-586.

[8] Nguyen, U. T. and Xu, J. "Multicast routing in wireless mesh networks: Minimum cost trees or shortest path trees?" In: IEEE Communications Magazine 45.11 (2007), pp. 7277.

[9] Gong, H., Fu, L., Fu, X., Zhao, L., Wang, K., and Wang, X. "Distributed multicast tree construction in wireless sensor networks". In: IEEE Transactions on Information Theory 63.1 (2016), pp. 280-296.

[10] Levin, D. "The environment constrains successful search strategies in natural distributed systems". In: (2016).

[11] Wiles, T. J., Jemielita, M., Baker, R. P., Schlomann, B. H., Logan, S. L., Ganz, J., Melancon, E., Eisen, J. S., Guillemin, K., and Parthasarathy, R. "Host gut motility promotes competitive exclusion within a model intestinal microbiota". In: PLoS biology 14.7 (2016), e1002517.

[12] Hein, A. M., Brumley, D. R., Carrara, F., Stocker, R., and Levin, S. A. "Physical limits on bacterial navigation in dynamic environments". In: J R Soc Interface 13.114 (2016), p. 20150844.

[13] Couzin, I. D., Krause, J., Franks, N. R., and Levin, S. A. "Effective leadership and decision-making in animal groups on the move". In: Nature 433.7025 (2005), p. 513.

[14] Gordon, D. M. “The ecology of collective behavior". In: PLoS Biol 12.3 (2014), e1001805.

[15] Gordon, D. M. "The evolution of the algorithms for collective behavior". In: Cell Syst 3.6 (2016), pp. 514-520. 
[16] Dussutour, A., Fourcassie, V., Helbing, D., and Deneubourg, J.-L. "Optimal traffic organization in ants under crowded conditions". In: Nature 428.6978 (2004), pp. 70-73.

[17] Latty, T., Ramsch, K., Ito, K., Nakagaki, T., Sumpter, D. J., Middendorf, M., and Beekman, M. "Structure and formation of ant transportation networks". In: J R Soc Interface 8.62 (2011), pp. 1298-1306.

[18] Middleton, E. J. and Latty, T. "Resilience in social insect infrastructure systems". In: Journal of The Royal Society Interface 13.116 (2016), p. 20151022.

[19] Gordon, D. M. "The ecology of collective behavior in ants". In: Annual review of entomology 64 (2019), pp. 35-50.

[20] Perna, A. and Latty, T. "Animal transportation networks". In: Journal of The Royal Society Interface 11.100 (2014), p. 20140334.

[21] Colorni, A., Dorigo, M., Maniezzo, V., et al. "Distributed optimization by ant colonies". In: Proceedings of the first European conference on artificial life. Vol. 142. Paris, France. 1991, pp. 134-142.

[22] Dorigo, M. and Blum, C. "Ant colony optimization theory: A survey". In: Theoretical Computer Science 344.2-3 (2005), pp. 243-278.

[23] López-Ibánez, M, Stützle, T, and Dorigo, M. “Ant Colony Optimization: A ComponentWise Overview”. In: (2015).

[24] Di Caro, G. and Dorigo, M. "AntNet: Distributed stigmergetic control for communications networks”. In: Journal of Artificial Intelligence Research 9 (1998), pp. 317-365.

[25] Gambardella, L. M., Montemanni, R., and Weyland, D. "Coupling ant colony systems with strong local searches". In: European Journal of Operational Research 220.3 (2012), pp. 831-843.

[26] Tsutsui, S. “Ant colony optimization with cunning ants". In: Transactions of the Japanese Society for Artificial Intelligence 22 (2007), pp. 29-36.

[27] Wiesemann, W. and Stützle, T. "Iterated ants: An experimental study for the quadratic assignment problem". In: International Workshop on Ant Colony Optimization and Swarm Intelligence. Springer. 2006, pp. 179-190.

[28] Malíčková, M., Yates, C., and Bodová, K. “A stochastic model of ant trail following with two pheromones". In: arXiv:1508.06816 (2015).

[29] Flanagan, T. P., Pinter-Wollman, N. M., Moses, M. E., and Gordon, D. M. "Fast and flexible: Argentine ants recruit from nearby trails". In: PloS one 8.8 (2013), e70888.

[30] Garnier, S., Guérécheau, A., Combe, M., Fourcassié, V., and Theraulaz, G. "Path selection and foraging efficiency in Argentine ant transport networks". In: Behavioral Ecology and Sociobiology 63.8 (2009), pp. 1167-1179.

[31] Deneubourg, J.-L., Goss, S., Franks, N., and Pasteels, J. "The blind leading the blind: modeling chemically mediated army ant raid patterns". In: Journal of insect behavior 2.5 (1989), pp. 719-725.

[32] Couzin, I. D. and Franks, N. R. "Self-organized lane formation and optimized traffic flow in army ants". In: Proceedings of the Royal Society of London. Series B: Biological Sciences 270.1511 (2003), pp. 139-146. 
[33] Cherix, D., Werner, P., Catzeflis, F, et al. "Spatial organisation of a polycalic system in Formica (Coptoformica) exsecta Nyl.(Hymenoptera: Formicidae)." In: Mitteilungen der Schweizerischen Entomologischen Gesellschaft 53.2/3 (1980), pp. 163-172.

[34] Cabanes, G., Wilgenburg, E. van, Beekman, M., and Latty, T. "Ants build transportation networks that optimize cost and efficiency at the expense of robustness". In: Behavioral Ecology 26.1 (2014), pp. 223-231.

[35] Bottinelli, A., Wilgenburg, E van, Sumpter, D. J., and Latty, T. "Local cost minimization in ant transport networks: from small-scale data to large-scale trade-offs". In: Journal of the Royal Society Interface 12.112 (2015), p. 20150780.

[36] Deneubourg, J. L., Aron, S., Goss, S., Pasteels, J., and Duerinck, G. "Random behaviour, amplification processes and number of participants: how they contribute to the foraging properties of ants". In: Physica D: Nonlinear Phenomena 22.1 (1986), pp. 176-186.

[37] Franks, N. R. “Army ants: a collective intelligence”. In: American Scientist 77 (1989), pp. 138-145.

[38] Bouchebti, S., Travaglini, R. V., Forti, L. C., and Fourcassié, V. "Dynamics of physical trail construction and of trail usage in the leaf-cutting ant Atta laevigata". In: Ethology Ecology \& Evolution 31.2 (2019), pp. 105-120.

[39] Countryman, S. M., Stumpe, M. C., Crow, S. P., Adler, F. R., Greene, M. J., Vonshak, M., and Gordon, D. M. "Collective search by ants in microgravity". In: Frontiers in Ecology and Evolution 3 (2015), p. 25.

[40] Garnier, S., Combe, M., Jost, C., and Theraulaz, G. "Do ants need to estimate the geometrical properties of trail bifurcations to find an efficient route? A swarm robotics test bed". In: PLoS computational biology 9.3 (2013), e1002903.

[41] Cook, Z., Franks, D. W., and Robinson, E. J. "Efficiency and robustness of ant colony transportation networks". In: Behavioral ecology and sociobiology 68.3 (2014), pp. 509517.

[42] Aron, S, Pasteels, J. M., and Deneubourg, J. L. "Trail-laying behaviour during exploratory recruitment in the argentine ant, Iridomyrmex humilis (Mayr)". In: Biology of Behaviour 14 (1989), pp. 207-217.

[43] Buhl, J., Hicks, K., Miller, E. R., Persey, S., Alinvi, O., and Sumpter, D. J. "Shape and efficiency of wood ant foraging networks". In: Behavioral Ecology and Sociobiology 63.3 (2009), pp. 451-460.

[44] Prömel, H. J. and Steger, A. The Steiner tree problem: a tour through graphs, algorithms, and complexity. Springer Science \& Business Media, 2012.

[45] Reid, C. R., Lutz, M. J., Powell, S., Kao, A. B., Couzin, I. D., and Garnier, S. “Army ants dynamically adjust living bridges in response to a cost-benefit trade-off". In: Proceedings of the National Academy of Sciences 112.49 (2015), pp. 15113-15118.

[46] Bles, O., Boehly, T., Deneubourg, J.-L., and Nicolis, S. C. "Same length, different shapes: ants collectively choose a straight foraging path over a bent one". In: Biology letters 14.3 (2018), p. 20180070. 
[47] Powell, S., Costa, A. N., Lopes, C. T., and Vasconcelos, H. L. "Canopy connectivity and the availability of diverse nesting resources affect species coexistence in arboreal ants". In: Journal of Animal Ecology 80.2 (2011), pp. 352-360.

[48] Gordon, D. M. "The dynamics of foraging trails in the tropical arboreal ant Cephalotes goniodontus". In: PLoS ONE 7.11 (2012), e50472.

[49] Gordon, D. M. "Local regulation of trail networks of the arboreal turtle ant, Cephalotes goniodontus". In: The American Naturalist 190.6 (2017), E156-E169.

[50] Dorigo, M. and Stützle, T. Ant Colony Optimization. Cambridge, MA: MIT Press, 2004.

[51] Eberhart, R. C., Kennedy, J., et al. "A new optimizer using particle swarm theory". In: Proceedings of the sixth international symposium on micro machine and human science. Vol. 1. New York, NY. 1995, pp. 39-43.

[52] Karaboga, D. An idea based on honey bee swarm for numerical optimization. Tech. rep. Technical report-tr06, Erciyes university, engineering faculty, computer engineering department, 2005.

[53] Chandrasekhar, A., Gordon, D. M., and Navlakha, S. "A distributed algorithm to maintain and repair the trail networks of arboreal ants". In: Sci Rep 8.1 (2018), p. 9297.

[54] Duan, X., George, M., Patel, R., and Bullo, F. "Robotic Surveillance Based on the Meeting Time of Random Walks”. In: arXiv preprint arXiv:1912.02693 (2019).

[55] Conover, W. and Iman, R. L. "Some exact tables for the squared ranks test". In: Communications in Statistics-Simulation and Computation 7.5 (1978), pp. 491-513.

[56] Dinno, A. conover.test: Conover-Iman Test of Multiple Comparisons Using Rank Sums. $\mathrm{R}$ package version 1.1.5. 2017.

[57] Sumpter, D. J. and Beekman, M. "From nonlinearity to optimality: pheromone trail foraging by ants". In: Animal behaviour 66.2 (2003), pp. 273-280.

[58] Emek, Y., Langner, T., Stolz, D., Uitto, J., and Wattenhofer, R. "How many ants does it take to find the food?" In: Theoretical Computer Science 608 (2015), pp. 255-267.

[59] Feinerman, O. and Korman, A. "The ANTS problem". In: Distributed Computing 30.3 (2017), pp. 149-168.

[60] Stickland, T., Britton, N. F., and Franks, N. R. "Complex trails and simple algorithms in ant foraging". In: Proceedings of the Royal Society of London. Series B: Biological Sciences 260.1357 (1995), pp. 53-58.

[61] Britton, N., Stickland, T., and Franks, N. "Analysis of ant foraging algorithms". In: Journal of Biological Systems 6.04 (1998), pp. 315-336.

[62] Monmarché, N., Venturini, G., and Slimane, M. "On how Pachycondyla apicalis ants suggest a new search algorithm". In: Future generation computer systems 16.8 (2000), pp. 937-946.

[63] Olson, M. E., Aguirre-Hernández, R., and Rosell, J. A. "Universal foliage-stem scaling across environments and species in dicot trees: plasticity, biomechanics and Corner's Rules”. In: Ecology Letters 12.3 (2009), pp. 210-219. 
[64] Byrka, J., Lewandowski, M., and Moldenhauer, C. "Approximation algorithms for nodeweighted prize-collecting Steiner tree problems on planar graphs". In: arXiv preprint arXiv:1601.02481 (2016).

[65] Bateni, M. H., Hajiaghayi, M. T., and Liaghat, V. "Improved Approximation Algorithms for (Budgeted) Node-weighted Steiner Problems". In: SIAM Journal on Computing 47.4 (2018), pp. 1275-1293.

[66] Yanoviak, S. and Kaspari, M. "Community structure and the habitat templet: ants in the tropical forest canopy and litter". In: Oikos 89.2 (2000), pp. 259-266.

[67] Vandermeer, J., Perfecto, I., and Philpott, S. M. "Clusters of ant colonies and robust criticality in a tropical agroecosystem”. In: Nature 451.7177 (2008), p. 457.

[68] Philpott, S. M., Perfecto, I., and Vandermeer, J. "Behavioral diversity of predatory arboreal ants in coffee agroecosystems". In: Environ. Entomol. 37.1 (2008), pp. 181-191.

[69] Jandt, J. and Gordon, D. "The behavioral ecology of variation in social insects". In: Current opinion in insect science 15 (2016), pp. 40-44.

[70] Werfel, J., Petersen, K., and Nagpal, R. "Designing collective behavior in a termiteinspired robot construction team”. In: Science 343.6172 (2014), pp. 754-758.

[71] Rees, W. M. van, Vouga, E., and Mahadevan, L. "Growth patterns for shape-shifting elastic bilayers". In: Proceedings of the National Academy of Sciences 114.44 (2017), pp. 11597-11602.

[72] Qi, L., Griego, A. D., Fricke, G. M., and Moses, M. E. "Comparing Physical and Simulated Performance of a Deterministic and a Bio-inspired Stochastic Foraging Strategy for Robot Swarms". In: Proceedings of the International Conference on Robotics and Automation (ICRA). IEEE. 2019.

[73] Bajaj, R. and Agrawal, D. P. "Improving scheduling of tasks in a heterogeneous environment". In: IEEE Transactions on Parallel and Distributed Systems 15.2 (2004), pp. 107118.

[74] Munguia, L.-M., Bader, D. A., and Ayguade, E. "Task-based parallel breadth-first search in heterogeneous environments". In: 2012 19th International Conference on High Performance Computing. IEEE. 2012, pp. 1-10.

[75] Pavone, M., Frazzoli, E., and Bullo, F. "Adaptive and distributed algorithms for vehicle routing in a stochastic and dynamic environment". In: IEEE Transactions on Automatic Control 56.6 (2010), pp. 1259-1274.

[76] Charikar, M., Chekuri, C., Cheung, T.-y., Dai, Z., Goel, A., Guha, S., and Li, M. "Approximation algorithms for directed Steiner problems". In: Journal of Algorithms 33.1 (1999), pp. 73-91.

[77] Scheirer, C. J., Ray, W. S., and Hare, N. "The analysis of ranked data derived from completely randomized factorial designs”. In: Biometrics (1976), pp. 429-434.

[78] Mangiafico, S. rcompanion: Functions to Support Extension Education Program Evaluation. $\mathrm{R}$ package version 2.3.7. 2019.

[79] Dreyfus, S. E. and Wagner, R. A. "The Steiner problem in graphs". In: Networks 1.3 (1971), pp. 195-207. 